\title{
The Existence of Positive Solutions of Singular Initial-Value Problem for Second Order Differential Equations-2
}

\author{
Afgan Aslanov ${ }^{1 *}$
}

\begin{abstract}
We consider the singular initial value problem for the second order differential equation. We interested in the existence of positive solutions and proved an easily applicable theorem on the existence of positive solutions to initial-value problems for second-order nonlinear singular differential equations. The previously established result on the existence of positive solution by Agarwal and O'Regan is not easy for the applications due to very complex definition of a new function and their properties in the statement of their theorem. We used the Lebesgue dominated convergence theorem and the Schauder-Tychonoff theorem in the proof of the main result. The main result can be easily applied for the singular and regular type of problems.

Keywords: Second order equations, Existence, Lane-Emden equation, Emden-Fowler equation, Fixed points 2010 AMS: $34 \mathrm{~A} 12$
\end{abstract}

\section{${ }^{1}$ Computer Engineering Department, Istanbul Esenyurt University, Istanbul, Turkey}

*Corresponding author: afganaslanov@yahoo.com

Received: 27 July 2020, Accepted: 14 December 2020, Available online: 22 December 2020

\section{Introduction}

In the mathematical literature there are many existence results on singular differential equations [3-8]. We are mainly motivated by [1] and the references therein.

We consider the problem

$$
\begin{aligned}
\left(p y^{\prime}\right)^{\prime}+p q g(y) & =0, t \in[0, T], \\
y(0) & =a>0, \\
\lim _{t \rightarrow 0+} p(t) y^{\prime}(t) & =0
\end{aligned}
$$

and

$$
\begin{aligned}
\left(p y^{\prime}\right)^{\prime}+p q g(y) & =0, t \in[0, T], \\
y(0) & =a>0, \\
y^{\prime}(0) & =0,
\end{aligned}
$$

where $0<T<\infty, p \geq 0, q \geq 0$ and $g:[0, \infty) \rightarrow[0, \infty)$. 
Agarwal and O'Regan [1] proved the next existence theorem for the positive solution of the problem (1.1) and (1.2).

Theorem 1.1. [1] Suppose the following conditions are satisfied

$$
\begin{aligned}
& p \in C[0, T) \cap C^{1}(0, T) \text { with } p>0 \text { on }(0, T), \\
& q \in L_{p}^{1}\left[0, t^{*}\right] \text { for any } t^{*} \in(0, T) \text { with } q>0 \text { on }(0, T) .
\end{aligned}
$$

Here $L_{r}^{1}[0, a]$ is the space of functions $u(t)$ with $\int_{0}^{a}|u(t)| r(t) d t<\infty$,

$$
\int_{0}^{t^{*}} \frac{1}{p(s)} \int_{0}^{s} p(x) q(x) d x d s<\infty \text { for any } t^{*} \in(0, T),
$$

and

$$
g:[0, \infty) \rightarrow[0, \infty) \text { is continuous, nondecreasing on }[0, \infty) \text { and } g(u)>0 \text { for } u>0 .
$$

Let

$$
H(z)=\int_{z}^{a} \frac{d x}{g(x)} \text { for } 0<z \leq a
$$

and

$$
\int_{0}^{t^{*}} \frac{1}{p(s)} \int_{0}^{s} p(x) q(x) \tau(x) d x d s<\text { a for any } t^{*} \in(0, T) .
$$

Here

$$
\tau(x)=g\left(H^{-1}\left(\int_{0}^{x} \frac{1}{p(w)} \int_{0}^{w} p(z) q(z) d z d w\right)\right) .
$$

Then equation (1) has a solution $y \in C[0, T)$ with $p y^{\prime} \in C[0, T),\left(p y^{\prime}\right)^{\prime} \in L_{p q}^{1}(0, T)$ and $0<y(t) \leq a$ for $t \in[0, T)$. In addition, if either

$$
p(0) \neq 0
$$

or

$$
p(0)=0 \text { and } \lim _{t \rightarrow 0+} \frac{p(t) q(t)}{p^{\prime}(t)}=0
$$

holds, then y is a solution of (1.2).

The condition (1.6) in connection with the definition of the function $\tau(x)$, makes this theorem difficult for application. In [2], we proved the theorem:

Theorem 1.2. Suppose (1.3)-(1.5) hold. In addition, we assume

$$
\begin{aligned}
\int_{0}^{t^{*}} \frac{1}{p(s)} \int_{0}^{s} p(x) q(x) g(a-\varphi(x)) d x d s & \leq a-\varphi(x), \\
\int_{0}^{t^{*}} \frac{1}{p(s)} \int_{0}^{s} p(x) q(x) g(\varphi(x)) d x d s & \geq \varphi(x)
\end{aligned}
$$

for some $\varphi(x) \in C[0, T]$, with $0 \leq \varphi(x) \leq a$. Then (1.1) has a solution $y \in C[0, T]$ with $p y^{\prime} \in C[0, T],\left(p y^{\prime}\right)^{\prime} \in L_{p q}^{1}(0, T)$ and $0<y(t) \leq$ a for $t \in[0, T]$.

The main purpose of this paper is to establish more easily applicable theorem on the existence of positive solution of the problem (1.1). 


\section{Main Results}

The following theorem is the main result of this article.

Theorem 2.1. Suppose the following conditions are satisfied

$$
\begin{aligned}
& p \in C[0, T) \cap C^{1}(0, T) \text { with } p>0 \text { on }(0, T], \\
& q \geq 0, \\
& \int_{0}^{t^{*}} \frac{1}{p(s)} \int_{0}^{s} p(x) q(x) d x d s<\infty \text { for any } t^{*} \in(0, T], \\
& g:[0, \infty) \rightarrow[0, \infty) \text { is continuous, nondecreasing on }[0, \infty),
\end{aligned}
$$

and assume

$$
\int_{0}^{t} \frac{1}{p(s)} \int_{0}^{s} p(x) q(x) g(a-\varphi(t)) d x d s \leq \varphi(t),
$$

for some $\varphi(t) \in C[0, T]$, with $0 \leq \varphi(t) \leq a, \varphi(0)=a$. Then equation (1) has a solution $y \in C[0, T]$ with py $\in C[0, T]$, $\left(p y^{\prime}\right)^{\prime} \in L_{p q}^{1}(0, T)$ and $0<y(t) \leq$ a for $t \in[0, T]$. In addition, if either

$$
p(0) \neq 0
$$

or

$$
p(0)=0 \text { and } \lim _{t \rightarrow 0+} \frac{p(t) q(t)}{p^{\prime}(t)}=0
$$

holds, then $y$ is a solution of (1.2).

Proof. We construct a sequence of functions such that the subsequences of odd-numbered terms and even-numbered terms are convergent. By using the limits of these sequences we construct a new set and the operator in this set. Then we use Schauder-Tychonoff theorem to show that this operator has a fixed point.

Consider the sequence $\left\{y_{n}(t)\right\}, n=0,1,2, \ldots$ with $y_{0}(t) \equiv \varphi(t)$,

$$
y_{n}(t)=\varphi(t)-\int_{0}^{t} \frac{1}{p(s)} \int_{0}^{s} p(x) q(x) g\left(y_{n-1}(x)\right) d x d s, n=1,2, \ldots, t \leq T .
$$

We have

$$
\begin{aligned}
& y_{0}(t) \equiv a-\varphi(t) \\
& y_{1}(t)=a-\int_{0}^{t} \frac{1}{p(s)} \int_{0}^{s} p(x) q(x) g\left(y_{0}(x)\right) d x d s \geq y_{0}(x),
\end{aligned}
$$

and in like manner

$$
\begin{aligned}
y_{2}(t) & =a-\int_{0}^{t} \frac{1}{p(s)} \int_{0}^{s} p(x) q(x) g\left(y_{1}(x)\right) d x d s \\
& \leq a-\int_{0}^{t} \frac{1}{p(s)} \int_{0}^{s} p(x) q(x) g\left(y_{0}(x)\right) d x d s \\
& =y_{1}(t), \\
y_{3}(t) & =a-\int_{0}^{t} \frac{1}{p(s)} \int_{0}^{s} p(x) q(x) g\left(y_{2}(x)\right) d x d s \geq y_{2}(t), \\
y_{4}(t) & =a-\int_{0}^{t} \frac{1}{p(s)} \int_{0}^{s} p(x) q(x) g\left(y_{3}(x)\right) d x d s \leq y_{3}(t), \ldots \\
y_{2 n-1}(t) & \geq y_{2 n-2}(t), \\
y_{2 n}(t) & \leq y_{2 n-1}(t), \ldots
\end{aligned}
$$


The sequences $\left\{y_{2 n}(t)\right\}$ and $\left\{y_{2 n+1}(t)\right\}$ are equicontinuous. Indeed, we have

$$
\left|y_{n}(t)-y_{n}(r)\right|=\int_{r}^{t} \frac{1}{p(s)} \int_{0}^{s} p(x) q(x) g\left(y_{n-1}(x)\right) d x d s \leq M \int_{r}^{t} \frac{1}{p(s)} \int_{0}^{s} p(x) q(x) d x d s
$$

where

$$
M=\max \{g(u): 0 \leq u \leq a\}
$$

and the right hand side of (2.1) can be taken $<\varepsilon$ for $|t-r|<\delta$, regardless of the choice of $t$ and $r$ : the function $\int_{0}^{t} \frac{1}{p(s)} \int_{0}^{s} p(x) q(x) d x d s$ is (uniformly) continuous on $[0, T]$. It follows from Ascoli Arzela Theorem that the sequence $\left\{y_{2 n}(t)\right\}$ has the (uniformly) convergent subsequence, $y_{2 n_{k}}(t) \rightarrow u(t)$. The Lebesgue dominated theorem guarantees that

$$
\begin{aligned}
y_{2 n_{k}+1}(t) & =a-\int_{0}^{t} \frac{1}{p(s)} \int_{0}^{s} p(x) q(x) g\left(y_{2 n_{k}}(x)\right) d x d s \rightarrow v(t), \\
v(t) & =a-\int_{0}^{t} \frac{1}{p(s)} \int_{0}^{s} p(x) q(x) g(u(x)) d x d s, \\
\text { and } u(t) & =a-\int_{0}^{t} \frac{1}{p(s)} \int_{0}^{s} p(x) q(x) g(v(x)) d x d s .
\end{aligned}
$$

If $u(t)=v(t)$; we have that the function $u(t)$ is the solution of the problem (1.1). Indeed, it follows from

$$
u(t)=a-\int_{0}^{t} \frac{1}{p(s)} \int_{0}^{s} p(x) q(x) g(u(x)) d x d s
$$

that

$$
\begin{aligned}
u^{\prime}(t) & =-\frac{1}{p(t)} \int_{0}^{t} p(x) q(x) g(u(x)) d x \\
p u^{\prime} & =-\int_{0}^{t} p(x) q(x) g(u(x)) d x \\
\left(p u^{\prime}\right)^{\prime} & =-p q g(u) .
\end{aligned}
$$

So, we suppose $u(t) \neq v(t)$. We have $u(0)=v(0)=a$ and if for example, $u(t)>v(t)$ on the interval $(0, b)$, then we obtain

$$
u(b)-v(b)=\int_{0}^{b} \frac{1}{p(s)} \int_{0}^{s} p(x) q(x)[g(u(x))-g(v(x)] d x d s>0
$$

and therefore $u(t)>v(t)$ on the whole interval $(0, T]$. The same holds for all points of intersections $t_{0}: u\left(t_{0}\right)=v\left(t_{0}\right)$. That is if $u\left(t_{0}\right)=v\left(t_{0}\right)$, then for any $\varepsilon>0$ there are infinitely many points $t_{n} \in\left[t_{0}, t_{0}+\varepsilon\right)$ such that $u\left(t_{n}\right)=v\left(t_{n}\right)$. Therefore, $u(t)>v(t)$ (or $i$ ) on $\left(t_{0}, T\right]$. Without loss of generality, let us suppose $u(t)>v(t)$ on $(0, T]$ and consider the operator $N: C[0, T] \rightarrow C[0, T]$ defined by

$$
N y(t)=a-\int_{0}^{t} \frac{1}{p(s)} \int_{0}^{s} p(x) q(x) g(y(x)) d x d s .
$$

Next, let

$$
K=\{y \in C[0, T]: v(t) \leq y(t) \leq u(t) \text { for } t \in[0, T]\} .
$$

The set $K$ is closed, convex and bounded subset of $C[0, T]$ and clearly $N: K \rightarrow K$. Let us show that $N: K \rightarrow K$ is continuous and compact operator. Continuity follows from Lebesgue dominated convergence theorem: if $y_{n}(t) \rightarrow y(t)$, then $N y_{n}(t) \rightarrow N y(t)$. To show that $N$ is completely continuous let $y(t) \in K$, then

$$
|N y(t)-N y(r)| \leq M\left|\int_{r}^{t} \frac{1}{p(x)} \int_{0}^{x} p(z) q(z) d z d s\right| \text { for } t, r \in[0, T]
$$


that is $N$ completely continuous on $[0, T]$. It follows from Schauder-Tychonoff theorem that $N$ has a fixed point $w \in K$, i.e. $w$ is a solution of (1.1). It follows from

$$
w^{\prime}(t)=-\frac{1}{p(t)} \int_{0}^{t} p(x) q(x) g(w(x)) d x
$$

that if $p(0) \neq 0$; then $w^{\prime}(0)=0$. Now if $p(0)=0$ but $\lim _{t \rightarrow 0+} \frac{p(t) q(t)}{p^{\prime}(t)}=0$ using L'Hôpital's rule we obtain from (8)

$$
\begin{aligned}
w^{\prime}(0+) & =-\lim _{t \rightarrow 0+} \frac{\int_{0}^{t} p(x) q(x) g(w(x)) d x}{p(t)} \\
& =-\lim _{t \rightarrow 0+} \frac{p(t) q(t) g(w(t))}{p^{\prime}(t)}=0,
\end{aligned}
$$

that is $w$ is a solution of (1.2). The proof is complete.

\section{Conclusion}

The theorem in [1] seems difficult for applications. The condition (1.6) very restrictive and decreases the sphere of applicability of the Theorem 1.1. We do not require the existence of the inverse $H^{-1}$. We proved an easily applicable theorem on the existence of positive solutions to initial-value problems for second-order nonlinear singular differential equations. The main result can be easily applied for the singular and regular type of problems.

\section{Conflict of interests}

The author declares that there is no conflict of interests regarding the publication of this paper.

\section{References}

[1] R. P. Agarwal, D. O'Regan, Second order initial value problems of Lane-Emden type, Appl. Math. Lett., 20 (2007), 1198-1205.

[2] A. Aslanov, A new theorem on the existence of positive solutions of singular initial-value problem for second order differential equations, Commun. Adv. Math. Sci., 2(1) (2019), 22-26.

[3] A. Constantin, On the existence of positive solutions of second-order differential equations, Ann. Mat. Pura Appl., 184(2005), 131-138.

[4] T. Ertem, A. Zafer, Asymptotic integration of second-order nonlinear differential equations via principal and nonprincipal solutions, Appl. Math. Comput., 219 (2013), 5876-5886.

[5] T. Ertem, A. Zafer, Existence of solutions for a class of nonlinear boundary value problems on half-line, Bound. Value Probl., 2012(2012), Article Number 43.

[6] T. Ertem, A. Zafer, Monotone positive solutions for a class of second-order nonlinear differential equations, . J. Comput. Appl. Math., 259(2014), 672-681.

[7] Z. Yin, Monotone positive solutions of second-order nonlinear differential equations, Nonlinear Anal., 54 (2003), $391-403$.

[8] Z. Zhao, Positive Solutions of Nonlinear Second Order Ordinary Differential Equations, Proc. Amer. Math. Soc., 121 (1994), 465-469. 